\title{
Factores socio-técnicos, modos de estudio en el continuo TIC-espacio- tiempo, y empleabilidad de estudiantes en una universidad online Socio-technical factors, way of studying in the ICT-space-time continuum, and students' employability in an online university
}

\author{
Juan-Francisco Martínez-Cerdá1 , Joan Torrent-Sellens ${ }^{2}$ \\ jmartinezcer@uoc.edu,jtorrent@uoc.edu \\ ${ }^{1}$ Estudios de Psicología y Ciencias de la Educación $\quad{ }^{2}$ Estudios de Economía y Empresa \\ Universitat Oberta de Catalunya \\ Barcelona, España
}

\begin{abstract}
Resumen- El aprendizaje a lo largo de la vida mediante educación online se ha ido consolidando como una metodología válida para la mejora de la empleabilidad. La investigación analiza las influencias que, sobre una empleabilidad diseñada de acuerdo a trece constructos latentes, tienen dos conjuntos de variables: 1) los cuatro factores socio-técnicos (STS) de una universidad online, con su orientación hacia: TIC, tareas para el aprendizaje, estudiantes, y el conjunto de la organización; y 2) las seis dimensiones relacionadas con el modo de estudio de sus estudiantes en el continuo espacio-tiempo conformado por la utilización de TIC para: prácticas pedagógicas, conectividad social, uso multitarea-policrónico (chronotopos), aprendizaje móvilininterrumpido (chronochora), uso equilibrado con la vida personal (kairochora), y uso tradicional-monocrónico (kairotopos). Aplicando modelización de rutas mediante mínimos cuadrados parciales (PLSPM) a datos primarios de una encuesta, se obtienen las siguientes conclusiones: i) tanto el sistema STS como el asociado al continuo TIC-espacio-tiempo influyen significativamente de un modo positivo sobre la empleabilidad; ii) los cuatro factores STS influyen significativamente de un modo positivo sobre el conjunto STS; y iii) las dimensiones 'uso equilibrado' y 'tradicional' no influyen significativamente sobre el sistema del continuo TIC-espacio-tiempo.
\end{abstract}

Palabras clave: Empleabilidad; educación online a lo largo de la vida; sistemas socio-técnicos; continuo TIC-espacio-tiempo; PLSPM

Abstract- Lifelong e-learning has been consolidated as a valid methodology for improving employability. Considering an employability based on thirteen latent constructs, the research analyzes the influences of two sets of variables on employability: 1) four socio-technical factors (STS) of an online university, according to its orientation towards: ICT, learning tasks, students, and the organization as a whole; and 2) six dimensions related to the elearners' way of studying in the space-time continuum and the use of ICT for: pedagogical practices, social connectivity, multi-taskingpolychronic use (chronotopos), mobile-seamless learning (chronochora), life-balanced use (kairochora), and traditionalmonocronic use (kairotopos). Applying partial least squares path modeling (PLS-PM) to survey primary data, the analysis obtain the following conclusions: i) both the STS system and the ITC-spacetime continuum have significant positive effects on employability; ii) the four STS factors have significant influences on the whole STS; and iii) the dimensions of 'balanced' and 'traditional' have not significant influences on the space-time continuum.

Keywords: Employability; Lifelong e-Learning; Socio-Technical Systems; ICT-Space-Time Continuum; PLS-PM

\section{INTRODUCCIÓN}

La economía del conocimiento y la globalización exige que los ciudadanos tengan que tener actualizadas sus competencias profesionales. Las nuevas aproximaciones para la organización del trabajo, basadas en un uso intensivo de las tecnologías de la información y la comunicación (TIC), están provocando el incremento de tareas no rutinarias (Autor, Levy, \& Murnane, 2003), hecho que genera que los trabajadores tengan que desarrollar numerosas destrezas de carácter avanzado (Spitz-Oener, 2006).

En este sentido, y tras una graduación universitaria que permite adquirir unas competencias con las que iniciar la carrera profesional (Michavila, Martínez, Martín-González, García-Peñalvo, \& Cruz-Benito, 2016), el aprendizaje a lo largo de la vida se configura como una de las más idóneas estrategias para el desarrollo de nuevas habilidades con las que poder hacer frente a las necesidades del mercado de trabajo (Morgan, Genre, \& Wilson, 2001). Así, este tipo de aprendizaje orientado hacia la empleabilidad va consolidándose paulatinamente (Bonacci, 2012), integrando las TIC, y convirtiéndose en aprendizaje a lo largo de la vida que es desarrollado mediante educación online por parte de numerosas instituciones (XiaoHai, 2010).

No obstante, su implementación encuentra diversos problemas. En el ámbito de la Unión Europea, a nivel político existen enfoques que diferencian entre estudiantes altamente competentes orientados hacia la 'economía' del conocimiento, frente a estudiantes menos competentes que tienen que hacer frente a una 'sociedad' del conocimiento (Brine, 2006). Además, también se observan barreras institucionales, situacionales, y de predisposición (Roosmaa \& Saar, 2016). Por otro lado, también existen diferencias culturales no subsanadas a escala global (Guo, 2010), que ofrecen un aprendizaje materialista orientado solamente hacia el mundo del trabajo (Jarvis, 2008), y que dibuja una educación meramente como producto de consumo y efímera (Bauman, 2003).

Desde esta perspectiva, la presente comunicación ofrece una visión de aprendizaje a lo largo de la vida que está orientada tanto a la empleabilidad de los ciudadanos como a su empoderamiento (Moir \& Crowther, 2014). En este sentido, cabe señalar la multitud de factores sociales (estudiantes en instituciones educativas) y técnicos (competencias a desarrollar en contextos tecnológicos) que intervienen en los 
procesos de aprendizaje online. Por ello, metodologías relacionadas con los sistemas socio-técnicos están siendo aplicadas al estudio del desarrollo de habilidades adquiridas mediante e-learning (Andrews \& Haythornthwaite, 2007; Upadhyaya \& Mallik, 2013).

Finalmente, diversas variables relacionadas con el espacio y el tiempo aparecen como constructos sociales de gran importancia en una educación en línea (Goodyear, 2006) en la que los estudiantes online manejan diversas escalas espacio temporales (Baldry \& Thibault, 2006). Por ello, la investigación tiene en cuenta factores relacionados con el modo de estudio de los estudiantes online, como elementos que afectan a los retos y procesos de reingeniería del elearning (Bernath, Szücs, Tait, \& Vidal, 2009; Varis \& Puukko, 2010).

\section{Contexto}

La presente comunicación tiene relación con la teoría del capital humano desarrollada durante la segunda mitad del siglo XX (Becker, 1993; Schultz, 1961). Esta teoría es utilizada por numerosas organizaciones e instituciones internacionales, como por ejemplo la OCDE (Rychen \& Salganik, 2005).

Concretamente, la aproximación considerada tiene en cuenta trece constructos latentes vinculados con habilidades para empleabilidad en el siglo XXI. Por un lado, cinco constructos relacionados con la disposición hacia la empleabilidad: apertura a los cambios (Lee, Song, Jeong, Lee, \& Yun, 2016), resiliencia/superación de adversidades (de Hoyos et al., 2013), proactividad (Lo Presti \& Pluviano, 2016), motivación por la carrera profesional (Van der Heijde, 2014), e identidad con el trabajo (Fugate \& Kinicki, 2008).
Por otro lado, ocho constructos del mercado de trabajo del siglo XXI: futuro de la securidad de puestos de trabajo (Calzada, 2005), futuro de la cultura en las organizaciones (Bollier, 2011; Castells, 2009), competencias colaborativas (Cedefop, 2015), competencias de alfabetización (OECD, 2013), competencias digitales (van Laar, van Deursen, van Dijk, \& de Haan, 2017), empoderamiento mediático (Martínez-Cerdá \& Torrent-Sellens, 2017), conversión del conocimiento (Martínez-León \& Ruiz Mercader, 2002), y adquisición del conocimiento (Torrent-Sellens, 2015).

La investigación también enlaza con la teoría de sistemas socio-técnicos (STS) desarrollada por Trist (1981) durante el mismo período. Esta teoría está siendo aplicada en contextos educativos (Checkland, 1981) y de aprendizaje a distancia (Bernath, Szücs, Tait, \& Vidal, 2009; Moore \& Kearsley 2011; Wang, Solan, \& Ghods, 2010). La teoría STS comprende dos subsistemas que interactúan conjuntamente: el subsistema social, relacionado con los estudiantes y la organización, y el subsistema técnico, vinculado a tareas y tecnologías (Emery, 1959). Esta aproximación permite considerar a las universidades online como un todo global formado por estos dos subsistemas que actúan conjuntamente de un modo complementario (Leavitt, 1965).

Finalmente, la investigación también conecta con las aproximaciones teóricas a los estilos de aprendizaje (Coffield, Moseley, Hall, \& Ecclestone, 2004), que tanta influencia tienen en la educación online desarrollada en entornos autoregulados (Zimmerman \& Martinez-Pons, 1988). Algunos de estos estilos están basados en el procesado de información (activo/reflexivo), percepción (sensorial/intuitivo), recepción de la información (visual/verbal) y entendimiento de la información (secuencial/global) (Graf, Liu, Kinshuk, Chen, \& Yang, 2009; Klašnja-Milićević, Vesin, Ivanović, \& Budimac, 2011; Özpolat, \& Akar, 2009).

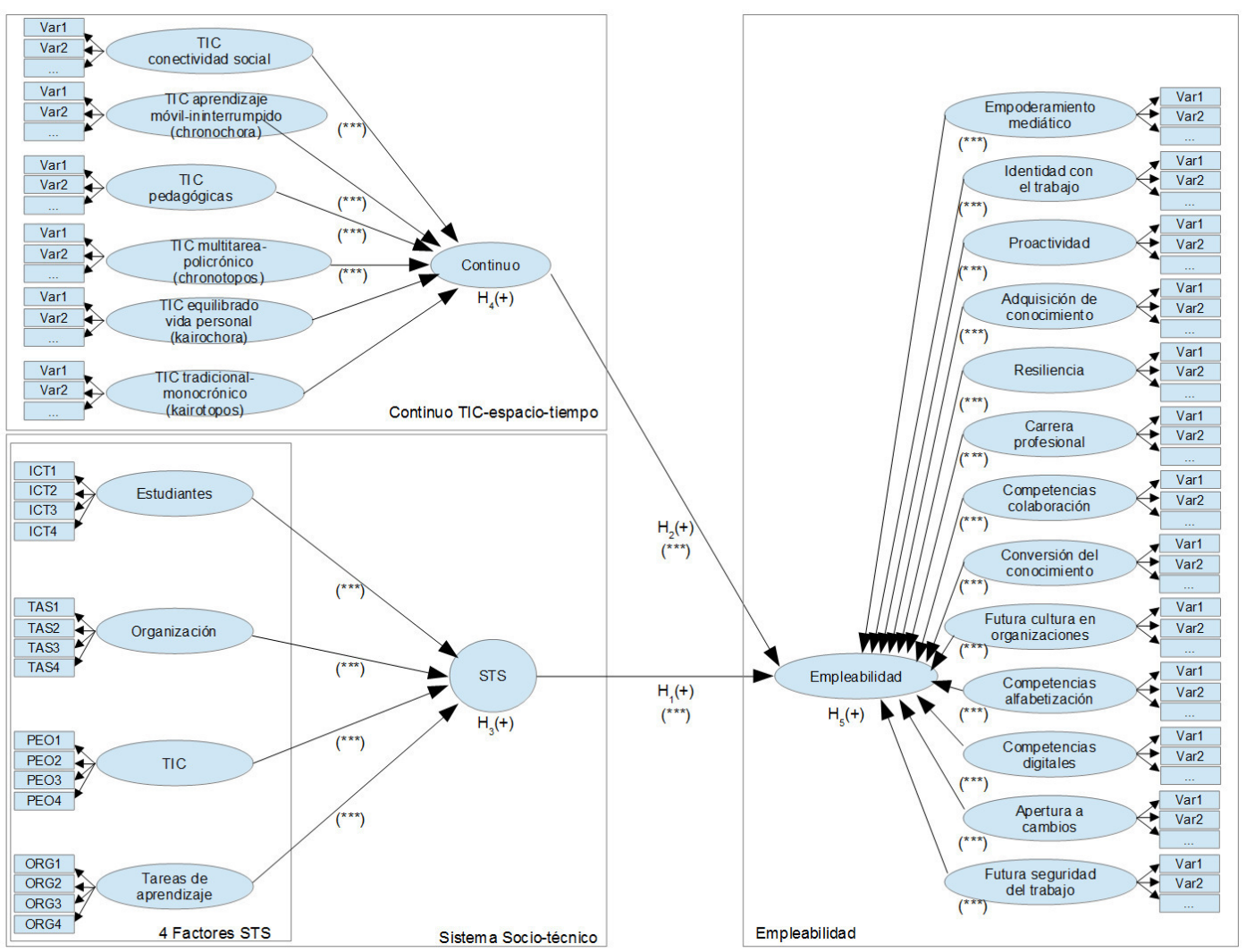

Figura 1: Framework para modelo de empleabilidad en sistema socio-técnico de universidad online y modos de estudio en el continuo TIC-espacio-tiempo 
No obstante, la aproximación llevada a cabo aquí tiene en cuenta el enfoque socio-técnico para la educación online y sus relaciones con TIC para el desarrollo de prácticas pedagógicas (Arribillaga, 2008; Sharpe, 2014), conectividad social (Cela, Sicilia, \& Sánchez, 2015; Wahlstedt, Pekkola, \& Niemelä, 2008), y el continuo espacio-tiempo planteado por los conceptos chronos-kairos y topos-chora (Capdeferro, Romero, \& Barberà Gregori, 2014; Lenhart, 2011; Rämö, 1999; Wong, 2012; Wong \& Looi, 2011).

De este modo, se definen seis factores que conforman el denominado continuo TIC-espacio-tiempo: i) TIC pedagógicas; ii) TIC para la conectividad social; iii) TIC con uso multitarea-policrónico (chronotopos); iv) TIC para aprendizaje móvil-ininterrumpido (chronochora); v) TIC bajo un uso equilibrado con la vida personal (kairochora); y vi) TIC con un uso tradicional-monocrónico (kairotopos).

La Figura 1 muestra el framework para modelo de empleabilidad en sistema socio-técnico de universidad online $\mathrm{y}$ modos de estudio en el continuo TIC-espacio-tiempo. Asimismo, también expone las hipótesis de investigación:

1. El conjunto del sistema socio-técnico (STS) en una universidad online tiene una influencia significativa positiva sobre el desarrollo de habilidades asociadas a la empleabilidad.

2. El conjunto del continuo TIC-espacio-tiempo tiene una influencia significativa positiva sobre el desarrollo de competencias vinculadas con la empleabilidad.

3. Los cuatro factores independientes del STS influyen significativamente de un modo positivo sobre el conjunto STS.

4. Las dimensiones independientes que forman el continuo TIC-espacio-tiempo tienen una influencia significativa positiva sobre dicho sistema, a excepción del 'uso equilibrado con la vida personal' y el 'uso tradicional-monocrónico'.

5. Las dimensiones consideradas para la empleabilidad influyen significativamente de un modo positivo sobre el conjunto global de empleabilidad.

\section{DESCRIPCIÓN}

Se realizó una búsqueda bibliográfica con la que definir el conjunto de ítems que serviría para el diseño del cuestionario que sería utilizado en una encuesta online. La Tabla 1 muestra el número de ítems y la gran cantidad de referencias y autores localizados para las dimensiones planteadas. Concretamente, el conjunto de ítems sirvió para crear un catálogo de especificaciones (Rust \& Golombok, 1999) que fue contrastado y validado externamente con una profesora de la Universitat de Barcelona. De este modo, se llevó a cabo una encuesta piloto con estudiantes de la Universitat Oberta de Catalunya (UOC). Tras su análisis y validación, se definió la versión definitiva del cuestionario online que fue utilizado durante el trabajo de campo, realizado con Alumni de la UOC.

El trabajo de campo fue realizado durante los meses de mayo y junio de 2016, durante los que se recibieron 550 respuestas válidas, conforme a un muestreo aleatorio no probabilístico. La tasa de respuesta fue del 1,0\%, que fue contrastada con recientes investigaciones llevadas a cabo también con cuestionarios online enviados a antiguos alumnos (Head, 2016; Wotherspoon \& McCarthy, 2016). El margen de error fue $\pm 4,18 \%$ para datos globales, población infinita, máxima indeterminación ( $\mathrm{p}=\mathrm{q}=50 \%$ ), y nivel de confianza de $95,5 \%$. Un $49,5 \%$ de la muestra fueron mujeres y un $50,5 \%$ fueron hombres, con una edad media de 46 años.

\section{Tabla 1}

Dimensiones, items y referencias utilizadas para la definición del cuestionario

\begin{tabular}{|c|c|c|}
\hline Dimensiones & $\begin{array}{l}\text { Número } \\
\text { de ítems }\end{array}$ & $\begin{array}{c}\text { Referencias } \\
\text { utilizadas }\end{array}$ \\
\hline \multicolumn{3}{|l|}{ Sistema socio-técnico } \\
\hline Estudiantes & 4 & 13 \\
\hline Organización & 4 & 10 \\
\hline TIC & 4 & 8 \\
\hline Tareas aprendizaje & 4 & 7 \\
\hline \multicolumn{3}{|c|}{ Continuo TIC-espacio-tiempo } \\
\hline TIC para la conectividad social & 3 & 13 \\
\hline TIC móvil-ininterrumpido (chronochora) & 3 & 7 \\
\hline TIC pedagógicas & 5 & 6 \\
\hline TIC multitarea-policrónico (chronotopos) & 3 & 10 \\
\hline TIC equilibrado vida personal (kairochora) & 3 & 12 \\
\hline TIC tradicional-monocrónico (kairotopos) & 3 & 7 \\
\hline \multicolumn{3}{|l|}{ Empleabilidad } \\
\hline Empoderamiento mediático & 8 & 19 \\
\hline Identidad con el trabajo & 5 & 14 \\
\hline Proactividad & 8 & 17 \\
\hline Adquisición del conocimiento & 6 & 17 \\
\hline Resiliencia/Superación de adversidades & 6 & 20 \\
\hline Motivación carrera profesional & 6 & 18 \\
\hline Competencias colaborativas & 4 & 9 \\
\hline Conversión del conocimiento & 4 & 9 \\
\hline Futuro de la cultura en las organizaciones & 6 & 11 \\
\hline Competencias de alfabetización & 3 & 4 \\
\hline Competencias digitales & 3 & 12 \\
\hline Apertura a los cambios & 4 & 14 \\
\hline Futuro de securidad de puestos de trabajo & 3 & 7 \\
\hline
\end{tabular}

Para el análisis de los datos se utilizó software diverso: IBM SPSS Statistics for Windows Version 22.0, R version 3.4.0, lavaan 0.5-23, y plspm 0.4.9. Conforme a las dimensiones identificadas a nivel teórico, se llevó a cabo un análisis factorial confirmatorio $(n=550)$, observando que se cumplieran diversos requerimientos relacionados con el mínimo número de individuos a analizar: 150 sujetos para análisis factoriales (Guadagnoli \& Velicer, 1988; Muthén \& Muthén, 2002), población igual o superior a 300 personas, y submuestra de modelo teórico igual o superior a 200 (Myers, Ahn \& Jin, 2011; Rouquette \& Falissard, 2011).

El análisis factorial confirmatorio utilizó el estimador DWLS para las escalas Likert 1-5 de las preguntas del cuestionario, y obtuvo los siguientes estadísticos $(\mathrm{N}=550)$ para el conjunto de constructos latentes señalados en la Figura 1: Minimum Function Test Statistic=20.210,564; Grado de libertad $=5.023 ; \quad$ P-value $\quad($ Chi-square $)=0.000 ; \quad \mathrm{CFI}=0,985$; TLI $=0,984 ; \quad$ IFI $=0,985 ; \quad$ RNI $=0,985 ; \quad$ RMSEA $=0,074 ; \quad y$ $\mathrm{SRMR}=0,069$. De este modo, las variables constructos latentes fueron confirmadas metodológicamente.

\section{Resultados}

La Tabla 2 muestra los resultados sintéticos obtenidos tras la aplicación de modelización de rutas mediante mínimos cuadrados parciales (PLS-PM) al conjunto de datos 
considerados $\quad\left(\right.$ bootstrap $=500 ; \quad \mathrm{GOF}=0,668 ; \quad \mathrm{R}^{2}(\mathrm{STS})=0,78$; $\mathrm{R}^{2}($ Continuo $)=0,51 ; \mathrm{R}^{2}($ Empleabilidad $\left.)=0,95\right)$.

\section{Tabla 2}

Variables, coeficientes estimados, y p-value del modelo de empleabilidad. Obtenido mediante PLS-PM

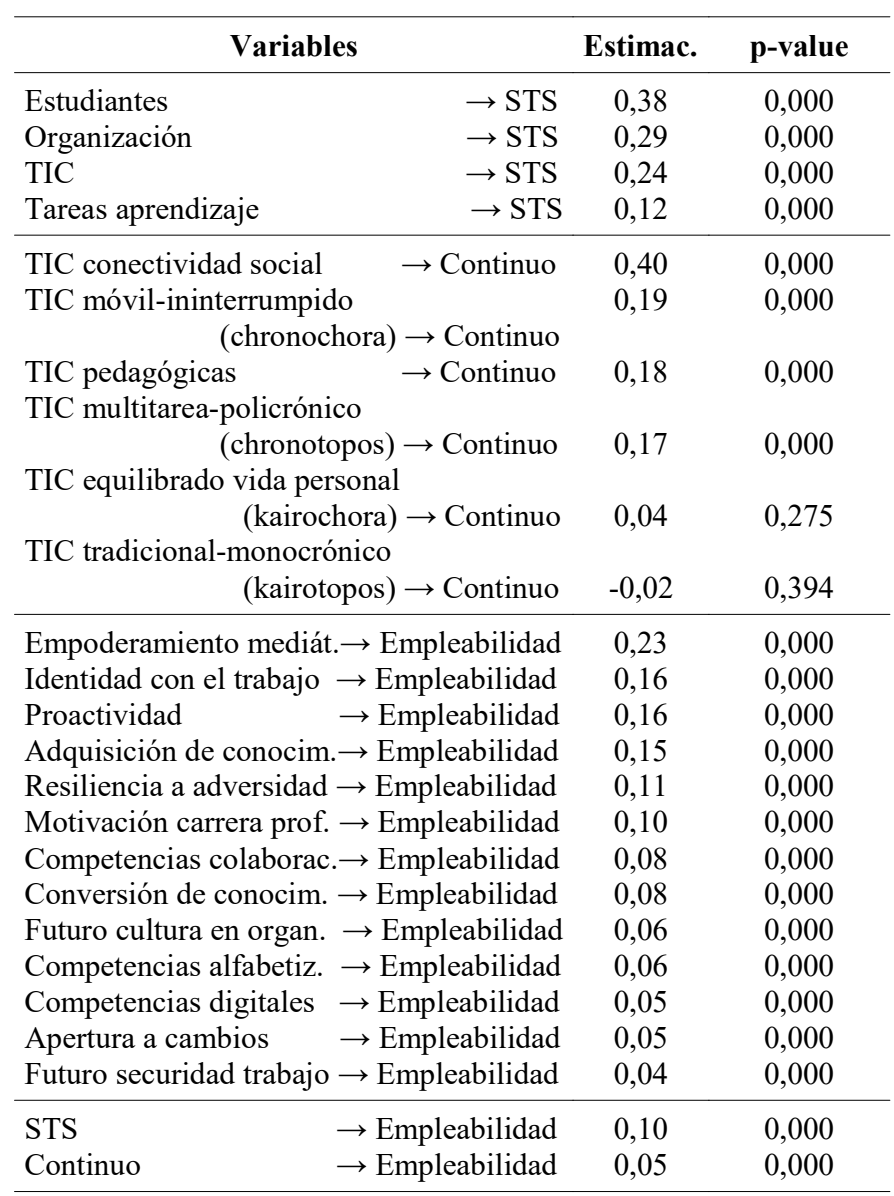

En general, se observa que el sistema STS influye significativamente $(B=0,10 ; p=0,000)$ sobre el constructo global de la empleabilidad. Lo mismo ocurre con el constructo relacionado con el uso de TIC en el continuo espacio-tiempo, que tiene también una influencia positiva, aunque de menor importancia $(B=0,05 ; p=0,000)$. Con ello de confirman las hipótesis 1 y 2 planteadas anteriormente.

En relación a los cuatro factores STS, el análisis realizado muestra que todos ellos influyen significativamente sobre el conjunto del sistema socio-técnico. El subsistema social, con orientación hacia los estudiantes $(\mathrm{B}=0,38)$ y cultura organizativa $(B=0,29)$, tiene mayor importancia que el subsistema técnico, formado por las TIC $(B=0,24)$ y las tareas para el aprendizaje $(B=0,12 ; \quad p=0,000$ en todos los constructos). Así, la hipótesis 3 queda también validada.

Respecto a las seis dimensiones de uso de las TIC en el continuo espacio-tiempo, se observa que dos constructos no influyen significativamente sobre tal continuo, confirmando la hipótesis 4 de la investigación: TIC equilibrado vida personal (kairochora) $(\mathrm{B}=0,04 ; \quad \mathrm{p}=0,275)$ y TIC tradicionalmonocrónico (kairotopos) $(\mathrm{B}=-0,02 ; \mathrm{p}=0,394)$. Respecto a los otros cuatro constructos, se detecta su influencia significativa, y la gran importancia de la conectividad social $(B=0,40)$ frente a la influencia similar de los otros tres constructos: TIC para aprendizaje móvil-ininterrumpido (chronochora), TIC para prácticas pedagógicas, y TIC para multitarea-policrónico (chronotopos) $(\mathrm{B}=0,19 ; \mathrm{B}=0,18 ; \mathrm{B}=0,17$; respectivamente; $\mathrm{y}$ $\mathrm{p}=0,000$ en todos los casos).

Finalmente, se observa que los trece constructos considerados para definir la empleabilidad influyen significativamente de un modo positivo sobre la empleabilidad, confirmando la hipótesis 5. Específicamente, se detecta la gran importancia del empoderamiento mediático $(B=0,23)$. Otras variables alcanzan valores relevantes, aunque inferiores: identidad y proactividad con el trabajo $(B=0,16$ en ambos casos), adquisición de conocimiento $(B=0,15)$, resiliencia $(\mathrm{B}=0,11)$, motivación por la carrera profesional $(B=0,10)$, competencias colaborativas $(B=0,08)$, y conversión del conocimiento $(\mathrm{B}=0,08)$. El resto de coeficientes oscila en la horquilla: $0,04-0,06(\mathrm{p}=0,000$ en todos los casos).

\section{Conclusiones}

La investigación realiza un análisis de diversos factores que influyen en el desarrollo de competencias relacionadas con la empleabilidad. Concretamente, de la influencia del sistema socio-técnico existente en una universidad online, y del modo de utilización de las TIC por parte de sus estudiantes en el continuo espacio-tiempo. El planteamiento de variables y constructos latentes es validado mediante análisis confirmatorio. La realización de modelización de rutas mediante mínimos cuadrados parciales (PLS-PM) permite obtener unos resultados que muestran, de cara a su influencia sobre el desarrollo de habilidades para la empleabilidad, la gran importancia de los factores sociales, del uso abstracto del tiempo por parte de los estudiantes online, y de la utilización de TIC para prácticas pedagógicas. Además, se observa la gran importancia del empoderamiento mediático como elemento para el desarrollo personal y profesional, junto a la disposición hacia la empleabilidad. Estas conclusiones deben ser tenidas en cuenta en organismos encargados del desarrollo de habilidades orientadas hacia la empleabilidad, en contextos educativos online para el aprendizaje a lo largo de la vida.

\section{Agradecimientos}

Juan-Francisco Martínez-Cerdá agradece el apoyo de una beca de doctorado de la Universitat Oberta de Catalunya.

\section{REFERENCIAS}

Andrews, R., \& Haythornthwaite, C. (Eds.). (2007). The SAGE Handbook of E-learning Research. London; Thousand Oaks: SAGE Publications.

Arribillaga, I. E. (2008). Active Knowledge Generation by University Students through Cooperative Learning. In ITI 6th International Conference on Information Communications Technology, 2008. ICICT 2008 (pp. 27-32).

Autor, D. H., Levy, F., \& Murnane, R. J. (2003). The Skill Content of Recent Technological Change: An Empirical Exploration. Quarterly Journal of Economics, 118(4), 1279-1333.

Baldry, A., \& Thibault, P. J. (2006). Multimodal Transcription and Text Analysis: a Multimodal Toolkit and Coursebook with Associated on-line Course. London: Equinox. 
Bauman, Z. (2003). Educational Challenges of the LiquidModern Era. Diogenes, 50(1), 15-26.

Becker, G.-S. (1993). Human Capital: a Theoretical and Empirical Analysis, with Special Reference to Education (Third Edition). New York: National Bureau of Economic Research: Distributed by Columbia University Press.

Bernath, U., Szücs, A., Tait, A., \& Vidal, M. (Eds.). (2009). Distance and e-learning in transition: learning innovation, technology and social challenges. Hoboken, NJ: ISTE/John Wiley.

Bollier, D. (2011). The future of work: what it means for individuals, businesses, markets and governments. Washington, DC: The Aspen Institute.

Bonacci, M. (2012). Experience Networking in the TVET System to Improve Occupational Competencies. ELearning Papers, 31, 18.

Brine, J. (2006). Lifelong learning and the knowledge economy: those that know and those that do not-the discourse of the European Union. British Educational Research Journal, 32(5), 649-665.

Calzada, I. (2005). Futuro del trabajo. Trabajo del futuro. Arrasate, Gipuzkoa: MIK.

Capdeferro, N., Romero, M., \& Barberà Gregori, E. (2014). Polychronicity: review of the literature and a new configuration for the study of this hidden dimension of online learning. Distance Education, 35(3), 294-310.

Castells, M. (2009). The Network Enterprise: The Culture, Institutions, and Organizations of the Informational Economy. In The Rise of the Network Society (pp. 163215). Wiley-Blackwell.

Cedefop. (2015). Skills, Qualifications and Jobs in the EU: the Making of a Perfect Match? Luxembourg: Publications Office of the European Union.

Cela, K. L., Sicilia, M. Á., \& Sánchez, S. (2015). Social Network Analysis in E-Learning Environments: A Preliminary Systematic Review. Educational Psychology Review, 27(1), 219-246.

Checkland, P. (1981). Systems Thinking, Systems Practice. Chichester, UK: Wiley.

Coffield, F., Moseley, D., Hall, E., \& Ecclestone, K. (2004). Learning styles and pedagogy in post-16 learning: $a$ systematic and critical review. London: Learning and Skills Research Centre.

De Hoyos, M., Green, A. E., Barnes, S.-A., Behle, H., Baldauf, B., Owen, D., \& Institute for Prospective Technological Studies. (2013). Literature Review on Employability, Inclusion and ICT, Report 2: ICT and Employability. (C. Centeno \& J. Stewart, Eds.) (Joint Research Centre of the European Commission). Luxembourg: Publications Office of the European Union.

Emery, F. E. (1959). Characteristics of socio-technical systems. In L. E. Davis \& J. C. Taylor (Eds.), Design of jobs: selected readings (pp. 177-198). Harmondsworth, UK: Penguin Books.
Fugate, M., \& Kinicki, A. J. (2008). A dispositional approach to employability: Development of a measure and test of implications for employee reactions to organizational change. Journal of Occupational \& Organizational Psychology, 81(3), 503-527.

Goodyear, P. (2006). Technology and the articulation of vocational and academic interests: reflections on time, space and e-learning. Studies in Continuing Education, 28(2), 83-98.

Graf, S., Liu, T.-C., Kinshuk, Chen, N.-S., \& Yang, S. J. H. (2009). Learning styles and cognitive traits - Their relationship and its benefits in web-based educational systems. Computers in Human Behavior, 25(6), 12801289.

Guadagnoli, E., \& Velicer, W. F. (1988). Relation of Sample Size to the Stability of Component Patterns. Psychological Bulletin, 103(2), 265-275.

Guo, S. (2010). Toward recognitive justice: emerging trends and challenges in transnational migration and lifelong learning. International Journal of Lifelong Education, 29(2), 149-167.

Head, A. J. (2016). Staying Smart: How Today's Graduates Continue to Learn Once They Complete College (SSRN Scholarly Paper No. ID 2712329) (p. 112). Rochester, NY: Social Science Research Network.

Jarvis, P. (2008). Democracy, Lifelong Learning and the Learning Society. Active Citizenship in a Late Modern Age (Vol. 3). New York: Routledge.

Klašnja-Milićević, A., Vesin, B., Ivanović, M., \& Budimac, Z. (2011). E-Learning personalization based on hybrid recommendation strategy and learning style identification. Computers \& Education, 56(3), 885-899.

Leavitt, H. J. (1965). Applied Organizational Change in Industry: Structural, technological and humanistic approaches. In J. G. March (Ed.), Handbook of organizations (pp. 1144 - 1170). Chicago: Rand McNally.

Lee, S., Song, Y., Jeong, M., Lee, C., \& Yun, G. (2016). Differences in the Influence of College Students' SelfEsteem for Getting-a-Job on Job Search Behavior Intensity in South Korea. Indian Journal of Science and Technology, 9(35).

Lenhart, I. (2011). Kairotopos: A reflection on Greek space/time concepts as design implications in Minecraft. In Proceedings of the 2011 DiGRA International Conference: Think Design Play (Vol. 6, pp. 1-18). Utrecht: Digital Games Research Association (DiGRA).

Lo Presti, A., \& Pluviano, S. (2016). Looking for a route in turbulent waters: Employability as a compass for career success. Organizational Psychology Review, 6(2), 192211.

Martínez-Cerdá, J.-F., \& Torrent-Sellens, J. (2017). Empoderamiento mediático mediante e-learning. Diseño y validación de una escala. El profesional de la información (EPI), 26(1), 43-54.

Martínez-León, I. M., \& Ruiz Mercader, J. (2002). Los procesos de creación del conocimiento: el aprendizaje y 
la espiral de conversión del conocimiento. In XVI Congreso Nacional y XII Congreso hispano-francés de AEDEM: la empresa intangible (pp. 1-16). Alicante: Asociación Europea de Dirección y Economía de la Empresa (AEDEM). Universidad de Alicante.

Michavila, F., Martínez, J. M., Martín-González, M., GarcíaPeñalvo, F. J., \& Cruz-Benito, J. (2016). Barómetro de empleabilidad y empleo de los universitarios en España, 2015 (Primer informe de resultados). Madrid: Observatorio de Empleabilidad y Empleo Universitarios.

Moir, S., \& Crowther, J. (2014). Learning for employability? Ideas to reassert a critical education practice in communities. Cuestiones Pedagógicas, 23, 43-64.

Moore, M. G., \& Kearsley, G. (2011). Distance education: a systems view of online learning. Belmont, CA: Wadsworth Cengage Learning.

Morgan, J., Genre, V., \& Wilson, C. (2001). Measuring Employment Security in Europe Using Surveys of Employers. Industrial Relations, 40(1), 54-72.

Muthén, L. K., \& Muthén, B. O. (2002). How to Use a Monte Carlo Study to Decide on Sample Size and Determine Power. Structural Equation Modeling: A Multidisciplinary Journal, 9(4), 599-620.

Myers, N. D., Ahn, S., \& Jin, Y. (2011). Sample Size and Power Estimates for a Confirmatory Factor Analytic Model in Exercise and Sport. Research Quarterly for Exercise and Sport, 82(3), 412-423.

OECD. (2013). OECD Skills Outlook 2013: First Results from the Survey of Adult Skills. Paris: OECD Publishing.

Özpolat, E., \& Akar, G. B. (2009). Automatic detection of learning styles for an e-learning system. Computers \& Education, 53(2), 355-367.

Rämö, H. (1999). An Aristotelian Human Time-Space Manifold From Chronochora to Kairotopos. Time \& Society, 8(2-3), 309-328.

Roosmaa, E.-L., \& Saar, E. (2016). Adults who do not want to participate in learning: a cross-national European analysis of their perceived barriers. International Journal of Lifelong Education, 0(0), 1-24.

Rouquette, A., \& Falissard, B. (2011). Sample Size Requirements for the Internal Validation of Psychiatric Scales. International Journal of Methods in Psychiatric Research, 20(4), 235-249.

Rust, J., \& Golombok, S. (1999). Modern Psychometrics: the Science of Psychological Assessment (Third). London; New York: Routledge.

Rychen, D. S., \& Salganik, L. H. (2005). The Definition and Selection of Key Competencies: Executive Summary (Definition and Selection of Competencies (DeSeCo)) (p. 20). Neuchâtel: Organisation for Economic Cooperation and Development (OECD).

Schultz, T.-W. (1961). Investment in Human Capital. The American Economic Review, 51(1), 1-17.

Sharpe, R. (2014). What does it take to learn in next generation learning spaces? In $\mathrm{K}$. Fraser (Ed.), The
Future of Learning and Teaching in Next Generation Learning Spaces (Vol. The Future of Learning and Teaching in Next Generation Learning Spaces, pp. 123145). Emerald Group Publishing Limited.

Spitz-Oener, A. (2006). Technical Change, Job Tasks, and Rising Educational Demands: Looking outside the Wage Structure. Journal of Labor Economics, 24(2), 235-270.

Torrent-Sellens, J. (2015). Knowledge Products and Network Externalities: Implications for the Business Strategy. Journal of the Knowledge Economy, 6(1), 138-156.

Trist, E. L. (1981). The Evolution of Socio-technical Systems: A Conceptual Framework and an Action Research Program. Toronto, Ontario: Ontario Ministry of Labour, Ontario Quality of Working Life Centre.

Upadhyaya, K. T., \& Mallik, D. (2013). E-Learning as a Socio-Technical System: An Insight into Factors Influencing its Effectiveness. Business Perspectives \& Research, 2(1), 1-12.

Van der Heijde, C. M. (2014). Employability and SelfRegulation in Contemporary Careers. In M. Coetzee (Ed.), Psycho-social Career Meta-capacities (pp. 7-17). Cham, Switzerland: Springer International Publishing.

Van Laar, E., Van Deursen, A. J. A. M., Van Dijk, J. A. G. M., \& De Haan, J. (2017). The relation between 21stcentury skills and digital skills: A systematic literature review. Computers in Human Behavior, 72, 577-588.

Varis, T., \& Puukko, M. (Eds.). (2010). Challenges of Global eLearning. Tampere: Tampere University, Research Centre for Vocational Education.

Wahlstedt, A., Pekkola, S., \& Niemelä, M. (2008). From elearning space to e-learning place. British Journal of Educational Technology, 39(6), 1020-1030.

Wang, J., Solan, D., \& Ghods, A. (2010). Distance learning success - a perspective from socio-technical systems theory. Behaviour \& Information Technology, 29(3), 321-329.

Wong, L.-H. (2012). A learner-centric view of mobile seamless learning. British Journal of Educational Technology, 43(1), E19-E23.

Wong, L.-H., \& Looi, C.-K. (2011). What seams do we remove in mobile-assisted seamless learning? A critical review of the literature. Computers \& Education, 57(4), 2364-2381.

Wotherspoon, S. E., \& McCarthy, P. W. (2016). The Factors and Motivations behind United Kingdom Chiropractic Professional Association Membership: a Survey of the Welsh Institute of Chiropractic Alumni. Chiropractic \& Manual Therapies, 24(35), 1-9.

XiaoHai, W. (2010). eLearning in Europe: Action plans and implications. In Artificial Intelligence and Education (ICAIE), 2010 International Conference on (pp. 240245). Hangzhou: IEEE.

Zimmerman, B. J., \& Martinez-Pons, M. (1988). Construct validation of a strategy model of student self-regulated learning. Journal of Educational Psychology, 80(3), 284-290. 